\title{
The Killer Immunoglobulin-Like Receptor (KIR) Group A Haplotype is Associated With Bronchiolitis Obliterans Syndrome After Lung Transplantation
}

Johanna M. Kwakkel-van Erp, MD, ${ }^{a}$ Ed A. van de Graaf, MD, PhD, ${ }^{\text {a }}$ Annelieke W. M. Paantjens, ${ }^{\mathrm{b}}$ Walter G. J. van Ginkel, ${ }^{\text {b }}$ Jennifer Schellekens, PhD, ${ }^{\text {b }}$ Diana A. van Kessel, MD, ${ }^{\mathrm{c}}$ Jules M. M. van den Bosch, $\mathrm{MD}, \mathrm{PhD},{ }^{\mathrm{c}}$ and Henny G. Otten, $\mathrm{PhD}^{\mathrm{b}}$

Background: The development of bronchiolitis obliterans syndrome (BOS) after lung transplantation is associated with viral infections. Natural killer (NK) cells are involved in the lysis of viral infected cells, and their activation is largely controlled by activating and inhibitory killer immunoglobulin-like receptors (KIRs). We hypothesized that KIR ligand incompatibility and recipients' individual KIRs could influence the development of BOS and the incidence of cytomegalovirus reactivation after lung transplantation.

Methods: $\quad$ The KIR gene contents were determined in 48 patients who received a lung transplant, and human leukocyte antigen (HLA)-Cw and HLA-Bw4 typing was performed on their respective donors.

Results: $\quad$ BOS developed in 7 patients and cytomegalovirus reactivation occurred in 16. BOS developed in 5 of 19 patients homozygous for KIR haplotype A compared with 2 of 27 patients with KIR haplotype $\mathrm{AB}$ and $\mathrm{B}$ (homozygous; $p=0.03$; log-rank test). In none of the patients with BOS was the activating KIR2DS5 gene detected, whereas it was present in $35 \%$ of patients without BOS $(p=0.04 ; \log$-rank test). No correlation was found between $K I R$ gene content and cytomegalovirus reactivation.

Conclusion: Our results suggest that the lack of activating KIRs may play an important role in the development of BOS but not in the control of cytomegalovirus reactivation after lung transplantation. J Heart Lung Transplant 2008;27:995-1001. Copyright (c) 2008 by the International Society for Heart and Lung Transplantation.

Lung transplantation is an ultimate therapy in patients with end-stage lung diseases who have no other therapeutic options left. Survival rates have improved over the years but are still restricted by the development of bronchiolitis obliterans syndrome (BOS). ${ }^{1}$ At present the exact mechanism of this chronic allograft rejection is not clear, but it has become evident that both innate and adaptive immune responses play an important role in the onset and pathogenesis of BOS. ${ }^{2-5}$

Natural killer (NK) cells are one of the main cellular components of the innate immune system and are important in the lysis of virally infected cells and

From the ${ }^{\mathrm{a}}$ Heart Lung Center Utrecht and ${ }^{\mathrm{b}}$ Department of Immunology, University Medical Center Utrecht, Utrecht; and ${ }^{\mathrm{c}}$ Heart Lung Center Utrecht, Saint Antonius Ziekenhuis, Nieuwegein, The Netherlands.

Submitted Feb 22, 2008; revised June 4, 2008; accepted June 10, 2008.

Reprint requests: H. G. Otten, Department of Immunology, UMC Utrecht, Postbox 85060, 3508 AB Utrecht, The Netherlands. Telephone: +31-88-7557680. Fax: +31-30-2521211. E-mail: h.g. otten@umcutrecht.nl

Copyright (c) 2008 by the International Society for Heart and Lung Transplantation. 1053-2498/08/\$-see front matter. doi:10.1016/ j.healun.2008.06.006 tumors. The NK cells express a multitude of intracellular and extracellular proteins in order to recognize and eliminate target cells. Recognition of target cells occurs through integration of different signals derived from cellsurface receptors, including major histocompatibility complex (MHC) class-I receptors (killer immunoglobulin-like receptors [KIRs], CD94/NKG2A, leukocyte immunoglobulin-like receptor, subfamily B1 [LLRB1]), adhesion molecules (CD2, CD11b/CD18, CD11c/CD18, CD31, CD96, CD49A/CD29, CD226), and costimulatory receptors (CD244 and NKG2D). ${ }^{6-8}$ Elimination of target cells expressing inadequate or no MHC class I molecules on their cell surface is regulated by opposite signals delivered by inhibitory and activating KIRs, of which the expression may be regulated depending on the type of NK-cell stimulus. ${ }^{9}$

KIRs are named according their structural and functional characteristics: the number of extracellular Ig domains (2D or 3D) and the intracellular tail (short [S] or long [L]). Short tails correlate with activating and long tails with inhibitory receptors. Although ligands for activating KIRs are still unknown, the inhibitory KIRs recognize epitopes on human leukocyte antigen (HLA)-C and HLA-Bw4. Ligands for KIR2DL1 are HLA-C with a lysine residue at position 80 (group 2), for 
KIR2DL2 and KIR2DL3, HLA-C with an asparagine residue at position 80 (group 1); and for KIR3DL1, HLA-Bw4.

The KIR genes are located on chromosome 19q13.4 within the leukocyte receptor complex (LRC). Based on population studies, multiple KIR haplotypes have been defined, differing in $K I R$ gene content and associated functional properties. ${ }^{10-12}$ The $K I R$ haplotype A contains 6 inhibitory and 1 activating $K I R$ genes, and expression of this haplotype will be associated with functional down-regulation of NK cell activity. ${ }^{13}$ The variety of $K I R$ haplotypes $\mathrm{B}$ all contain a mixture of functional activating and inhibiting KIRs. ${ }^{11,14}$

In previous reports on hematopoietic stem cell transplantation, significant associations were found between KIR genes (the presence of specific KIRs, the number of KIRs, mismatching of inhibitory KIRs with their respective HLA ligands) and clinical key parameters, including chronic graft-versus-host disease, which can manifest in the lungs as bronchiolitis obliterans and cytomegalovirus (CMV) reactivation. ${ }^{15-17}$

Recent data demonstrated that in patients with BOS, the number of peripheral blood NK cells is decreased but an increased number are present in transplanted lungs compared with stable patients. These data suggest that NK cells are recruited from blood to the lungs during the process of chronic rejection. ${ }^{18}$ However, the role of KIRs responsible for NK cell function after lung transplantation is not elucidated yet. In the present study, we examined patients' KIR gene content and hypothesized that KIR ligand incompatibility or $K I R$ haplotypes could be associated with the development of BOS or CMV reactivation, or both.

\section{PATIENTS AND METHODS Subjects}

The study included 48 patients who underwent lung transplantation at the Heart Lung Center in Utrecht between October 2001 and March 2006 and survived more than 3 months. To eliminate post-operative complications, we excluded patients who died during the period from transplantation until 3 months after transplantation. According to the standard International Society of Heart and Lung Transplantation criteria, BOS was defined as a decline of the forced expiratory volume in 1 second from the post-operative baseline at 2 distinctive time-points of more than 20\% in the absence of infection or other etiology. ${ }^{19}$ Patients were monitored for graft function and CMV infections.

Standard immunosuppressive therapy consisted of basiliximab as induction immunosuppression, tacrolimus, mycophenolate mofetil, and prednisone. Patients at risk for CMV reactivation, which is defined as a CMV seropositive donor or patient, were treated with valganciclovir for at least 6 months after transplantation. The study design was approved by the Medical Ethical Committee, and informed consent was obtained from each patient.

\section{Genotyping}

Genomic DNA from patients and donors was isolated from peripheral blood lymphocytes, stored, and retrospectively typed. Complementary DNA from donors was typed for HLA-B and HLA-C by polymerase chain reaction (PCR)-specific priming (SSP; Biotest, Dreieich, Germany). HLA-C and HLA-B were segregated into the epitopes groups HLA-C group 1 (HLA-C Asn 80: HLACw1,3,7,8,13,14 alleles), HLA-C group 2 (HLA-Clys80: HLA-Cw2,4,5,6,12,15,17,18 alleles), and HLA-Bw4. ${ }^{20}$ DNA from patients was typed for 6 activating $K I R$ genes (2DS1, 2DS2, 2DS3, 2DS4, 2DS5, and 3DS1) and for 8 inhibitory $K I R$ genes (2DL1, 2DL2, 2DL3, 2DL4, 2DL5, 3DL1, 3DL2, and 3DL3) by LUMINEX SSO (One Lambda Inc, Canoga Park, CA). A missing KIR ligand was defined as the presence of patients' KIR genes in the absence of their corresponding HLA on the transplanted lungs (ligand). The following KIR genes match with their ligands: $2 D L 1$ corresponds with HLA-C group 2, 2DL2/3 with HLA-C group 1, and 3DL1 with HLA-Bw4.

At the moment, there are 42 KIR haplotypes detected based on their gene content. All haplotypes contain the framework genes $3 D L 3,2 D L 4$, and $3 D L 2$ and contain either $2 D L 2$ or $2 D L 3$, but not both. ${ }^{10-12}$ Haplotype A contains framework genes and $2 D L 3,2 D L 1,3 D L 1$, and 2DS4. Haplotype B is more variable and contains more than one activating KIR gene. The presence of $K I R$ genes $2 D L 5,2 D S 1,2 D S 2,2 D S 3$, or $2 D S 5$ reflects haplotypes B. ${ }^{14}$ The presence of KIR haplotypes A (homozygous), $\mathrm{AB}$, and $\mathrm{B}$ (homozygous) in patients were deducted from the type and number of KIR genes detected. $^{10,12}$

\section{Latent CMV Reactivation}

The CMV serostatus of patients and donors was determined by enzyme-linked immunoassay (ELISA; VIDASbiomerieux, Marcy L'Etoile, France). We excluded patients with a negative CMV serostatus before transplantation who received lungs from CMV-negative donors. Monitoring after transplantation for CMV reactivation was performed by PCR. We used the PCR (Taqman) whereby a serologic reactivation was defined as a successive assay detecting more than 400 copies/ml in serum. After October 2003, we enlarged our input and used $3 \mathrm{ml}$ of serum instead of $1 \mathrm{ml}$; therefore, our threshold for a successive assay could be lowered to $50 \mathrm{CMV}$ copies $/ \mathrm{ml}$ instead of 400 copies $/ \mathrm{ml}$. In 6 patients, CMV copies were initially measured with a threshold of 400 copies $/ \mathrm{ml}$, and CMV reactivation occurred in 4 . Patients with a clinical CMV reactivation, defined as more than 1000 copies/ml were treated with 
valganciclovir and reducing immune suppression. Duration and intensity of valganciclovir depended on clinical status and viral load.

\section{Statistical Analysis}

The associations between patient characteristics and freedom from BOS and CMV reactivation were analyzed with a log-rank test in the Kaplan-Meier curve. The Fisher exact test was used to compare frequencies. A value of $p<0.05$ was considered statistically significant.

\section{RESULTS}

The median follow-up time of the 48 patients studied was 24 months (range, 9-60 months). All but 5 patients (all non-BOS) received a bilateral lung transplant. BOS developed in 7 patients. Two patients died during the course of the study, one of which was associated with BOS. The patients with BOS were similar to those without BOS in age, gender, and underlying disease. Baseline characteristics of the overall study group are summarized in Table 1.

\section{Relationship Between KIR-Ligand Matching and Development of BOS}

To examine whether the absence or presence of KIR ligands on transplanted lungs was associated with the development of BOS, we typed patients' KIR genes and HLA of the donor. KIR genotyping of the 48 patients revealed $47(98 \%)$ were positive for $2 D L 1$ and $44(92 \%)$ for $3 D L 1$. Forty patients (85\%) were positive for $K I R 2 D L 3$, and $1940(\%)$ were positive for KIR2DL2. DNA could not be obtained from 2 donors for HLA typing, but 38 donors (83\%) who could be analyzed were typed for HLA-C group 1, 32 (70\%) for HLA-C group 2, and 36 (75\%) were positive for HLA-Bw4.

An analysis of patients' KIR genes with the absence of the corresponding HLA ligands on the transplanted lungs found 28 lung transplants (58\%) had HLA ligand(s) missing for patients' KIRs. One KIR ligand was missing in $20(71 \%)$ patients, and 2 ligands were missing in 8 (29\%). The distribution of patients missing KIRs and HLA ligands is detailed in Table 2. No significant differences in freedom from BOS were found between patients with and without KIR ligands.

\section{Relationship Between Patients' KIRs and Development of BOS}

We also examined whether a relation exists between BOS and the number of activating or inhibitory KIRs in

Table 1. Baseline Characteristics of Overall Study Group

\begin{tabular}{|c|c|c|c|}
\hline Characteristics & All & BOS & Non-B0S \\
\hline Number of patients & 48 & 7 & 41 \\
\hline Median age, years (range) & $50(16-64)$ & $56(24-60)$ & $50(16-64)$ \\
\hline \multicolumn{4}{|l|}{ Underlying disease } \\
\hline - COPD & $25(52 \%)$ & $3(43 \%)$ & $22(54 \%)$ \\
\hline$-\mathrm{CF}$ & $13(27 \%)$ & $1(14 \%)$ & $12(29 \%)$ \\
\hline - interstitial lung disease & $10(21 \%)$ & $3(43 \%)$ & $7(17 \%)$ \\
\hline \multicolumn{4}{|l|}{ Gender } \\
\hline M & $23(48 \%)$ & $2(29 \%)$ & $21(51 \%)$ \\
\hline $\mathrm{F}$ & $25(52 \%)$ & $5(71 \%)$ & $20(49 \%)$ \\
\hline \multicolumn{4}{|l|}{ Recipient-KIR-haplotypes } \\
\hline - KIR haplotype A (homozygous) & $19(40 \%)$ & $5(71 \%)$ & $14(34 \%)$ \\
\hline - KIR haplotype A and B & $14(29 \%)$ & $1(14 \%)$ & $13(31 \%)$ \\
\hline - KIR haplotype B (homozygous) & $15(31 \%)$ & $1(14 \%)$ & $14(34 \%)$ \\
\hline Median number of activating KIR genes (range) & $3(1-6)$ & $1(1-5)$ & $3(1-6)$ \\
\hline Median number of inhibitory KIR genes (range) & $6(2-8)$ & $6(6-8)$ & $7(2-8)$ \\
\hline Median KIR mismatches (range) & $1(0-2)$ & $1(0-2)$ & $1(0-2)$ \\
\hline \multicolumn{4}{|l|}{ CMV status recipient/donor (pre transplant) } \\
\hline - positive/positive & $8(17 \%)$ & $2(29 \%)$ & $6(15 \%)$ \\
\hline - positive/negative & $15(31 \%)$ & 0 & $15(37 \%)$ \\
\hline - negative/positive & $12(25 \%)$ & $3(43 \%)$ & $9(22 \%)$ \\
\hline - negative/negative & $13(27 \%)$ & $2(29 \%)$ & $11(27 \%)$ \\
\hline CMV reactivation & $16 / 36$ & $3 / 5$ & $13 / 30$ \\
\hline \multicolumn{4}{|l|}{ Median HLA mismatches (range) } \\
\hline Class I & $3(2-4)$ & $3(2-4)$ & $3(2-4)$ \\
\hline Class II & $1(0-2)$ & $1(1-2)$ & $1(0-2)$ \\
\hline
\end{tabular}

Definition of abbreviations: COPD, chronic obstructive pulmonary disease; CF, Cystic Fibrosis; M, Male; F, Female; KIR, Killer Immunoglobulin-like Receptors; HLA, Human Leucocytes Antigen; CMV, cytomegalovirus. 
Table 2. Characteristics of Ligand Absence Between Transplanted Lungs and Kir Patient

\begin{tabular}{|c|c|c|}
\hline \multicolumn{3}{|c|}{ Ligand absent, number (\%) } \\
\hline Lung (donor) & Recipient & \\
\hline HLA-C group 1 (HLA-C $\left.{ }^{\text {Asn80 }}\right)$ & $\begin{array}{l}\text { absent for KIR 2DL2/2DL3 } \\
\text { patient }\end{array}$ & $10(22 \%)$ \\
\hline HLA-C group 2 (HLA-C ${ }^{\text {Lys80 }}$ ) & absent for KIR 2DL1 patient & $15(33 \%)$ \\
\hline HLA-Bw4 on & absent for KIR 3DL1 patient & $12(25 \%)$ \\
\hline HLA-Bw4 and HLA-C & absent for patient KIR & $8(17 \%)$ \\
\hline No ligand absence & & $9(19 \%)$ \\
\hline
\end{tabular}

Definition of abbreviations: KIR, Killer Immunoglobulin-like Receptors; HLA, Human Leucocytes Antigen.

individual patients. The median number of activating KIRs was 3 (range, $1-6$ ), and 13 patients (27\%) had 5 or more activating KIRs. The median number of inhibitory KIRs was 6 (range, 2-8), and 24 patients (50\%) had 7 or more inhibitory KIRs. No significant relation was found between the number of inhibitory or activating KIRs and the absence or presence of BOS.

Although some individual KIR genes (e.g., 2DL2, $2 D L 5,2 D S 1$, and 2DS2) appeared to differ in their frequencies in BOS patients compared with non-BOS patients, this did not reach statistical significance (Figure 1). The KIR2DS5 gene, however, was present in 14 non-BOS patients (34\%) but absent in patients with BOS, which was significantly associated with the development of BOS (hazard ratio, $0.00 ; p=0.04$; Figure 2).

\section{KIR Haplotypes and BOS}

Nineteen patients (40\%) were homozygous for $K I R$ haplotype A, 15 (31\%) had a copy of KIR haplotypes A and $\mathrm{B}$, and 14 (29\%) were homozygous for KIR haplotype B. No association was found between KIR haplo-

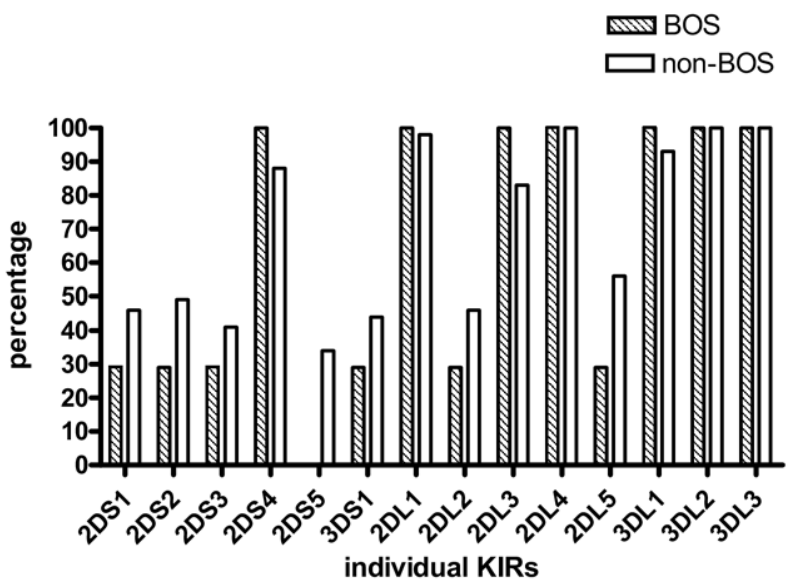

Figure 1. Frequency of individual killer immunoglobulin-like receptors (KIR) genes in patients with and without bronchiolitis obliterans syndrome (BOS) was determined as described in the Materials and Methods section. Shown are the frequencies of individual KIR genes found in patients with (gray bars) or without (white bars) BOS.

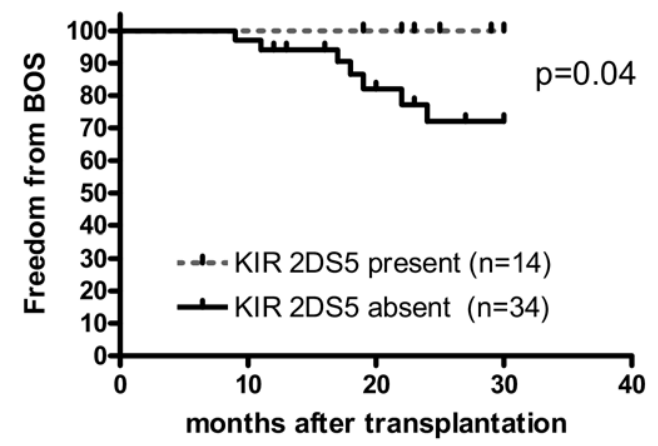

Figure 2. Kaplan-Meier analysis shows of freedom from bronchiolitis obliterans syndrome (BOS) was significantly different between the 34 patients with killer immunoglobulin-like receptor (KIR) gene 2DS5 absent (solid line) and the 14 patients with KIR2DS5 present (dashed line). Log-rank test, $p=0.04$.

types and underlying lung diseases. A comparison of KIR haplotypes between BOS and non-BOS patients found that $5(71 \%), 1(14 \%)$, and 1 patients (14\%) with BOS had KIR haplotypes $\mathrm{A}, \mathrm{AB}$, or $\mathrm{B}$, respectively, whereas $14(34 \%), 14(34 \%)$, and 13 (32\%) from the non-BOS patients contained these haplotypes. The presence of KIR haplotype A resulted in a significant higher risk of BOS development (hazard ratio, $4.9 ; p=0.03$; Figure 3).

\section{Influences of Missing KIR Ligands, Individual KIR Genes, and KIR Haplotypes on CMV Reactivation}

To examine whether CMV reactivation was related to KIR gene content, we studied CMV serostatus in donors and patients before transplantation and linked them to individual KIR genes. This analysis indicated that in 12 transplantations, CMV was neither present in donor nor patient. From the 36 remaining patients at risk for a CMV reactivation, a reactivation actually occurred in 16 patients despite prophylactic use of ganciclovir. CMV

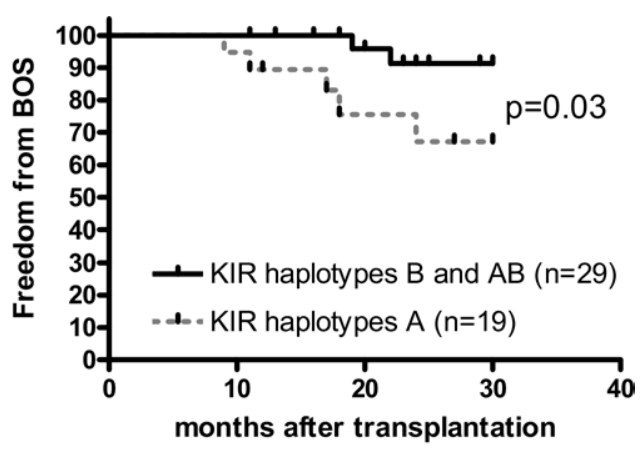

Figure 3. Influence of killer immunoglobulin-like receptor (KIR) haplotype A on freedom from bronchiolitis obliterans syndrome (BOS) by Kaplan-Meier analysis showed a significant difference between the 19 patients with KIR haplotype A (homozygous, dashed line) and the 29 patients with $K I R$ haplotype $A B$ and $B$ (homozygous, solid line). Log-rank test, $p=0.03$. 


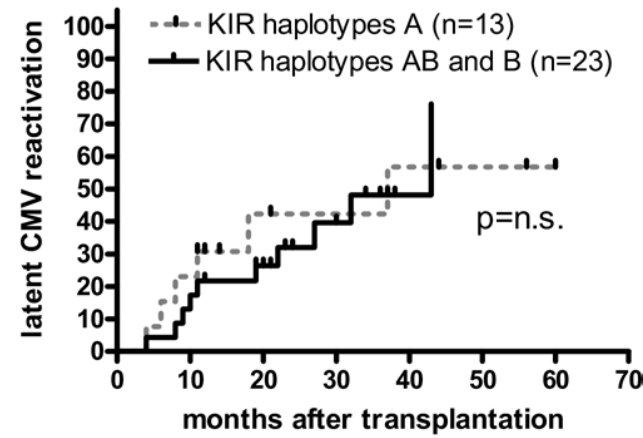

Figure 4. Kaplan-Meier analysis of cytomegalovirus (CMV) reactivation showed no significant difference between the 13 patients with killer immunoglobulin-like receptor (KIR) haplotype A (homozygous, dashed line) and the 23 patients with KIR haplotype $A B$ and $B$ (homozygous, solid line). CMV reaction was defined as a successive assay in the polymerase chain reaction after transplantation in those patients who had a seropositive CMV status before transplantation or who received lungs from a CMV seropositive donor.

reactivation occurred at a median of 11 months (range, 4-43 months) after transplantation.

KIR genotyping of the 36 patients at risk for CMV reactivation showed that 35 patients (97\%) were positive for KIR2DL1, all (100\%) for KIR2DL2 or KIR $2 D L 3$ (53\% for KIR2DL2, 86\% for KIR2DL3), and 34 (94\%) were positive for KIR3DL1. Eighteen of the transplanted lungs (69\%) were typed for HLA-C group 1, 27 (75\%) for HLA-C group 2, and 34 (94\%) for HLA-Bw4. Twenty-two patients with CMV reactivation received a lung with KIR ligands missing, whereas in the other 14 patients, all ligands were present on the transplanted lungs. Studying the influence of missing KIR ligands on CMV reactivation showed no significant differences in patients with or without a missing KIR ligand. In addition, no significant relation could be found between the number of inhibitory or activating KIR genes, neither between individual KIR genes nor CMV reactivation. Furthermore, no significant correlation was found between the KIR haplotypes of patients and CMV reactivation (Figure 4 ).

\section{DISCUSSION}

Stimulated by the reported associations between KIRs and several clinical features after hematopoietic stem cell transplantation, we hypothesized that KIR gene content may be related to chronic allograft rejection and CMV reactivation after lung transplantation. To our knowledge, this is the first study to analyze the influence of individual KIR gene contents, their corresponding ligands, and haplotypes on the results of lung transplantation. We demonstrate that KIR haplotype A and the absence of KIR2DS5, presumably the lack of activating KIRs, are predictive for development of chronic allograft rejection.
As inhibitory signals derived from KIRs are primarily regulated by ligation with their corresponding HLAligands, we first analyzed KIR2DL1, KIR2DL2, KIR2DL3, and KIR3DL1 from all patients, and HLA-Cw and HLABw4 in the donors. An effect of KIR-ligand matching on chronic rejection after lung transplantation was not detected. These data are in line with a previous report showing no effect of KIR ligand-matching on the 10year allograft survival in 2,757 patients receiving cadaver kidney transplantation. ${ }^{21}$ In a recent study of 69 kidney transplants, no association was found between individual KIR genes or KIR haplotypes and the occurrence of acute rejection when tapering their immunosuppressive regimen. ${ }^{22}$ However, Kunert et al ${ }^{23}$ demonstrated in 224 kidney transplants that patients without acute rejections had higher frequencies of KIR2DL2/KIR2DS2 although no association was found between acute rejections and KIR haplotypes.

In the present study we have found that both $K I R$ haplotype $\mathrm{A}$ and the absence of KIR2DS5 are significantly associated with BOS. This finding is unexpected when the viewpoint is taken that NK cells are simply killer cells guided by their cell-surface receptors. Because NK cells containing the homozygous KIR haplotype A express a phenotype mainly encoded by inhibitory KIRs, this KIR haplotype should be associated with less reactivity against donor cells recognized on lung allografts, and thus absence from BOS, instead of the association found in the present study. It is possible that the differences observed are a random effect due to a limited sample size. Nevertheless, if these findings are confirmed in a larger study, one could speculate that NK cells play a more regulatory role in the alloresponse (e.g., by dendritic cell editing) in the period of months and years after transplantation when allograft damage is persistently induced by the immune system. ${ }^{24}$ In the lung, immature dendritic cells are located in airway epithelium, alveolar septa, and around pulmonary vessels. These immature dendritic cells are sensitive to killing by NK cells, especially by those expressing activating KIRs and Toll-like receptors. When immature dendritic cells become activated and mature, they down-regulate secretion of chemokines (CCR1, CCR5, and CCR6), up-regulate expression of CCR $7,{ }^{25,26}$ and become insensitive to lysis by NK cells. ${ }^{27,28}$ The efficiency with which activated NK cells kill immature dendritic cells may differ between those containing the $K I R$ haplotype $\mathrm{A}$ vs $\mathrm{AB}$ or $\mathrm{B}$ haplotypes, which will result downstream in differences in alloantigen presentation of dendritic cells to $T$ cells.

A couple of arguments made the study analyzing the influence of NK cells on CMV reactivation are appealing. The first is that NK cells are important in the control of viral infection, and second is the association between viral infections, especially CMV, with the 
development of BOS. ${ }^{19,29}$ Studies in patients with human immunodeficiency virus have shown that the combination of the activating KIR3DS1 and its presumed ligand HLA-Bw4 is associated with a delayed progression to acquired immunodeficiency syndrome, whereas the presence of $3 D S 1$ in absence of $\mathrm{Bw} 4$ is associated with a rapid progression. These data suggest that virus elimination is facilitated upon binding of an activating KIR to its ligand. ${ }^{30}$

After hematopoietic stem cell transplantation, missing ligands for inhibitory KIRs did not influence CMV reactivation, but results of such studies in organ transplantation have not been reported yet. ${ }^{31}$ In our study on lung transplantation, we did not observe any relation between missing KIR ligands or in the number of activating KIRs or individual KIRs on CMV reactivation. After allogeneic stem cell transplantation, however, patients with $K I R$ haplotypes $\mathrm{AB}$ and $\mathrm{B}$-and thus with more activating KIRs-showed a lowered incidence of CMV reactivation compared with those with KIR group A haplotypes. ${ }^{16,31}$ Because CMV reactivation after lung transplantation is associated with BOS, and the KIR haplotypes $\mathrm{AB}$ and $\mathrm{B}$ are associated with lack of $\mathrm{BOS}$, we expected a protective influence of activating KIRs on CMV reactivation, but did not find any.

Next to this difference, CMV reactivation occurred 10 to 20 days after stem cell transplantation in $55 \%$ to $63 \%$ of patients vs 4 months after lung transplantation in $44 \%(n=16)$, despite prophylactic use of ganciclovir. Apparently, in the stem cell transplantation setting where myeloablative therapy was given and the immune system was reconstituting under immunosuppression, the influence of KIRs on CMV reactivation differs from that in organ transplantation in which a fully functional immune system is only reduced in activity by immunosuppressive therapy.

Our results show that the KIR gene content of recipients, especially activating KIRs, influences chronic allograft rejection after lung transplantation, possibly through editing of dendritic cells. We are aware that our study is hampered by the small patient population and a relatively short follow-up. Our findings need to be confirmed in larger studies elucidating the role of KIR usage by NK cells in chronic allograft rejection. We propose a detailed analysis of NK cells infiltrating lung allografts, including the influence of different immunosuppressives on their function. A challenging issue will be to further explore the mechanism of NK cells activation by characterizing activating ligands and their expression on the lung allograft.

\section{REFERENCES}

1. Trulock EP, Edwards LB, Taylor DO, Boucek MM, Keck BM, Hertz MI. Registry of the International Society for Heart and Lung Transplantation: Twenty-third official adult lung and heart-lung transplantation report-2006. J Heart Lung Transplant 2006; 25(8):880-92.

2. Terasaki PI. Humoral theory of transplantation. Am J Transplant 2003;3(6):665-73.

3. Gould DS, Auchincloss H. Direct and indirect recognition: the role of MHC antigens in graft rejection. Immunol Today 1999; 20(2):77-82.

4. Côté I, Rogers NJ, Lechler RI. Allorecognition. Transfus Clin Biol 2001;8(3):318-23.

5. Jiang S, Herrera O, Lechler RI. New spectrum of allorecognition pathways: implications for graft rejection and transplantation tolerance. Curr Opin Immunol 2004;16(5):550-7.

6. Middleton D, Curran M, Maxwell L. Natural killer cells and their receptors. Transplant Immunol 2002;10(2-3):147-64.

7. Parham P. MHC class I molecules and KIRs in human history, health and survival. Nat Rev Immunol 2005;5(3):201-14.

8. Anfossi N, Andre P, Guia S, Falk CS, et al. Human NK cell education by inhibitory receptors for MHC class I. Immunity 2006;25(2):331- 42 .

9. Huard B, Fruh K. A role for MHC class I down-regulation in NK cell lysis of herpes virus-infected cells. Eur J Immunol 2000;30(2) 509-15.

10. Hsu KC, Liu XR, Selvakumar A, Mickelson E, O'Reilly RJ, Dupont B. Killer Ig-like receptor haplotype analysis by gene content: Evidence for genomic diversity with a minimum of six basic framework haplotypes, each with multiple subsets. J Immunol 2002 Nov 1;169(9):5118-29.

11. Hsu KC, Chida S, Dupont B, Geraghty DE. The killer cell immunoglobulin-like receptor (KIR) genomic region: gene-order, haplotypes and allelic polymorphism. Immunol Rev 2002;190(1):40-52.

12. Uhrberg M, Parham P, Wernet P. Definition of gene content for nine common group $\mathrm{B}$ haplotypes of the Caucasoid population: KIR haplotypes contain between seven and eleven KIR genes. Immunogenetics 2002;54(4):221-9.

13. Kikuchi-Maki A, Yusa S, Catina TL, Campbell KS. KIR2DL4 is an IL-2-regulated NK cell receptor that exhibits limited expression in humans but triggers strong IFN-gamma production. J Immunol 2003 Oct 1;171(7):3415-25.

14. Marsh SG, Parham P, Dupont B, et al. Killer-cell immunoglobulinlike receptor (KIR) nomenclature report, 2002. Immunogenetics 2003;55(4):220-6

15. Kroger N, Binder T, Zabelina $\mathrm{T}$, et al. Low number of donor activating killer immunoglobulin-like receptors (KIR) genes but not KIR-ligand mismatch prevents relapse and improves disease-free survival in leukemia patients after in vivo T-Cell depleted unrelated stem cell transplantation. Transplantation 2006;82(8):1024-30.

16. Cook M, Briggs D, Craddock C, et al. Donor KIR genotype has a major influence on the rate of cytomegalovirus reactivation following T-cell replete stem cell transplantation. Blood 2006; 107(3):1230-2.

17. De Santis D, Bishara A, Witt CS, et al. Natural killer cell HLA-C epitopes and killer cell immunoglobulin-like receptors both influence outcome of mismatched unrelated donor bone marrow transplants. Tissue Antigens 2005;65(6):519-28.

18. Fildes JE, Yonan N, Tunstall $\mathrm{K}$, et al. Natural killer cells in peripheral blood and lung tissue are associated with chronic rejection after lung transplantation. J Heart Lung Transplant 2008;27(2):203-7.

19. Estenne M, Hertz MI. Bronchiolitis obliterans after human lung transplantation. Am J Respir Crit Care Med 2002;166(4): $440-4$.

20. Ruggeri L, Capanni M, Casucci M, et al. Role of natural killer cell alloreactivity in HLA-mismatched hematopoietic stem cell transplantation. Blood 1999;94(1):333-9. 
21. Tran TH, Mytilineos J, Scherer S, Laux G, Middleton D, Opelz G. Analysis of KIR ligand incompatibility in human renal transplantation. Transplantation 2005;80(8):1121-3.

22. Kreijveld E, Van der Meer A, Tijssen HJ, Hilbrands LB, Joosten I. KIR gene and KIR ligand analysis to predict graft rejection after renal transplantation. Transplantation 2007;84(8):1045-51.

23. Kunert K, Seiler M, Mashreghi MF, et al. KIR/HLA ligand incompatibility in kidney transplantation. Transplantation 2007;84(11): 1527-33.

24. Marcenaro E, Dondero A, Moretta A. Multi-directional crossregulation of NK cell function during innate immune responses. Transplant Immunol 2006;17(1):16-9.

25. Saeki H, Moore AM, Brown MJ, Hwang ST. Cutting edge: secondary lymphoid-tissue chemokine (SLC) and CC chemokine receptor 7 (CCR7) participate in the emigration pathway of mature dendritic cells from the skin to regional lymph nodes. J Immunol 1999; 162(5):2472-5.
26. Sallusto F, Palermo B, Lenig D, et al. Distinct patterns and kinetics of chemokine production regulate dendritic cell function. Eur J Immunol 1999;29(5):1617-25.

27. Holt PG, Haining S, Nelson DJ, Sedgwick JD. Origin and steady-state turnover of class-Ii Mhc-bearing dendritic cells in the epithelium of the conducting airways. J Immunol 1994;153(1):256-61.

28. Cooper MA, Fehniger TA, Fuchs A, Colonna M, Caligiuri MA. NK cell and DC interactions. Trends Immunol 2004;25(1):47-52.

29. Boehler A, Estenne M. Post-transplant bronchiolitis obliterans Eur Respir J 2003;22(6):1007-18.

30. Martin MP, Gao XJ, Lee JH, et al. Epistatic interaction between KIR3DS1 and HLA-B delays the progression to AIDS. Nat Genet 2002;31(4):429-34.

31. Chen C, Busson M, Rocha V, et al. Activating KIR genes are associated with CMV reactivation and survival after non-T-cell depleted HLA-identical sibling bone marrow transplantation for malignant disorders. Bone Marrow Transplant 2006;38(6):437-44. 